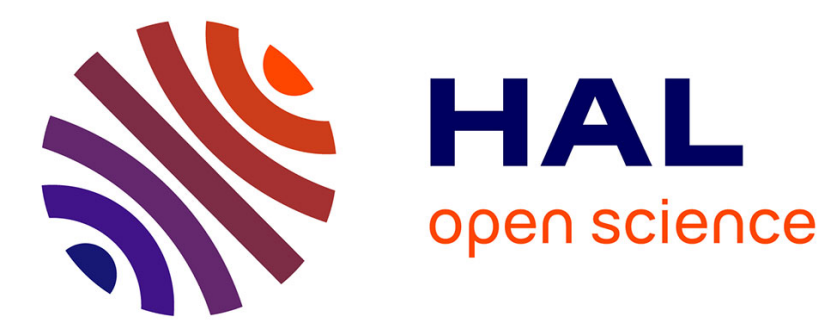

\title{
The Parr formula for the superheating field in a semi-infinite film
}

Pierre del Castillo

\section{To cite this version:}

Pierre del Castillo. The Parr formula for the superheating field in a semi-infinite film. Journal of Mathematical Physics, 2005, 46, pp.053513. 10.1063/1.1900292 . hal-00016502

\section{HAL Id: hal-00016502 https://hal.science/hal-00016502}

Submitted on 6 Jan 2006

HAL is a multi-disciplinary open access archive for the deposit and dissemination of scientific research documents, whether they are published or not. The documents may come from teaching and research institutions in France or abroad, or from public or private research centers.
L'archive ouverte pluridisciplinaire HAL, est destinée au dépôt et à la diffusion de documents scientifiques de niveau recherche, publiés ou non, émanant des établissements d'enseignement et de recherche français ou étrangers, des laboratoires publics ou privés. 


\title{
The Parr formula for the superheating field in a semi-infinite film
}

\author{
Pierre Del Castillo \\ Laboratoire Amiénois de Mathématiques Fondamentales et Appliquées, CNRS UMR 6140, \\ Université de Picardie Jules Verne, 33 r. Saint-Leu, 80039 Amiens Cedex 1, France \\ e-mail: pierre.delcastillo@u-picardie.fr
}

\begin{abstract}
In [16], Di Bartolo, Dolgert and Dorsey have constructed asymptotic matched solutions at order two for the half-space Ginzburg-Landau model in the weak- $\kappa$ limit. These authors deduced a formal expansion for the superheating field $h^{s h}(\kappa)$ up to order four, extending the de Gennes formula [17] and the two terms in Parr's formula [23]. On the other hand, we have obtained in [13] two terms in the lower bound for $h^{s h}(\kappa)$. In this paper, we prove rigorously that the second term of the expansion of $h^{s h}(\kappa)$ is of the order of $\mathcal{O}\left(\kappa^{\frac{1}{2}}\right)$ and we get the Parr formula. We improve the upper bound obtained by Bolley and Helffer in [5] and we get

$$
\kappa\left(h^{s h}(\kappa)\right)^{2} \leq 2^{-\frac{3}{2}}+\frac{15}{32} \kappa+\mathcal{O}\left(\kappa^{1+\rho}\right), \quad \rho>0
$$

The proof is based on new estimates for $f^{\prime}, A$ and $A^{\prime}$. To achieve this, we are guided by the analysis of the properties of the approximate solution constructed in [14]-[16].
\end{abstract}

\section{Introdution}

The states of a superconducting material in an exterior magnetic field are described by the GinzburgLandau theory which introduces a functional depending in particular on a complex wave function and on the magnetic potential $A$. These states are characterized as global or local minima of this functional. When the sample is a film and the exterior magnetic field is parallel to the surface, the Ginzburg-Landau model reduces to a one-dimensional problem where the wave function is real (and denoted by $f$ ) and where the functional is the following:

$$
\varepsilon_{d}(f, A ; h)=\int_{-\frac{d}{2}}^{\frac{d}{2}}\left[\frac{1}{2}\left(1-f(x)^{2}\right)^{2}-\frac{1}{2}+\kappa^{-2} f^{\prime}(x)^{2}+f(x)^{2} A(x)^{2}+\left(A^{\prime}(x)-h\right)^{2}\right] d x,
$$

with $(f, A) \in\left(H^{1}\left(\left[-\frac{d}{2}, \frac{d}{2}\right]\right)\right)^{2}$. Here, $d$ is proportional to the thickness of the film, $h$ is proportional to the exterior magnetic field and $\kappa$ is the Ginzburg-Landau parameter characterizing the properties of the material. The value of $\kappa$ determines the type of superconductor according to the type of phase transition which takes place between the normal phase and the superconducting phase. $\kappa$ small describes what is known as a type I superconductor and $\kappa$ large as a type II. More precisely, for a type I superconductor, there is a critical magnetic field $h_{c}$ such that is $h<h_{c}$, the material is entirely superconducting and the magnetic field is expelled from the sample apart from a boundary layer of size $\lambda$. This is called the Meissner effect. If $h>h_{c}$, superconductivity is destroyed and the material is in the normal state, that is $f \equiv 0$ and $A^{\prime} \equiv h$. For a type II superconductor, the phase transition 
is different and there are two critical fields $h_{c_{1}}$ and $h_{c_{2}}$. For $h<h_{c_{1}}$, the exterior magnetic field is expelled from the sample and there is a Meissner effect as for type I superconductors. When $h$ increases above $h_{c_{1}}$, superconductivity is not destroyed straight away, since the superconducting and the normal phase coexist under the form of filaments or vortices. As $h$ increases further, the vortices become more numerous until the critical value $h_{c_{2}}$ is reached at which superconductivity is destroyed. For $h>h_{c_{2}}$, the materiel is in the normal state. The way superconductivity is nucleated is highly dependent on $d$ and $\kappa$ (see for example [25]).

In the following, we restrict us to the research of symmetric solutions. By symmetric solutions, we mean solutions $(f, A)$ such that $f$ is even and $A$ is odd. Hence, we reduce the study of $\varepsilon_{d}$ to the interval $]-d, 0[$ and then to $] 0, d[$ by a translation (the edge of the film is then at 0 ). A reduced GL functional is then defined by

$$
\varepsilon_{d}(f, A ; h)=\int_{0}^{d}\left[\frac{1}{2} f^{4}-f^{2}+\kappa^{-2}\left(f^{\prime}\right)^{2}+f^{2} A^{2}+\left(A^{\prime}-h\right)^{2}\right] d x,
$$

for the pairs $(f, A)$ of $H^{1}(] 0, d[)^{2}$ such that $A(d)=0$.

When the width $d$ of the film is large (in the sense that $\kappa d$ is large), a slightly different modelization is considered, which was first introduced by Ginzburg and which is usually called the superconducting half-space. To get formally the limiting problem on the interval $[0,+\infty[$, we put $d=+\infty$ in the definition of the GL functional (1.1) after a renormalization obtained by adding the term $\left(h^{2}-\frac{1}{2}\right) d$. We then get

$$
\varepsilon_{\infty}(f, A)=\int_{0}^{+\infty}\left[\frac{1}{2}\left(1-f(x)^{2}\right)^{2}+\kappa^{-2} f^{\prime}(x)^{2}+f(x)^{2} A(x)^{2}+A^{\prime}(x)^{2}\right] d x+2 h A(0),
$$

defined for $(f, A) \in E_{\infty}=\left\{(f, A) ;(1-f) \in H^{1}(] 0,+\infty[), A \in H^{1}(] 0,+\infty[)\right\}$. The corresponding Ginzburg-Landau equations expressing the necessary conditions for minima are then

$$
(G L)_{\infty}\left\{\begin{array}{ccc}
(a)-\kappa^{-2} f^{\prime \prime}-f+f^{3}+f A^{2} & =0 & \text { on }[0,+\infty[ \\
(b) & -A^{\prime \prime}+A f^{2} & \text { on }[0,+\infty[
\end{array}\right.
$$

with the boundary conditions

$$
f^{\prime}(0)=0, \quad A^{\prime}(0)=h .
$$

The problem $(G L)_{\infty}$ is called the half-space model and was studied in [18] and [19] where numerical solutions are given.

We consider the set $\mathcal{H}_{\infty} \subset \mathbb{R}^{+}$of the $h$ 's such that there exist solutions of the (G.L.) system with $f>0$. We know that $\mathcal{H}_{\infty}$ is a bounded interval $\left[0, h^{+}\right.$) (see [3], Proposition 2.1) and we then introduce the superheating field $h^{\text {sh }}(\kappa)$, defined as the supremum of the interval $\mathcal{H}_{\infty}$. This critical field is very important for many applications. For instance, measuring the superheating field provides one of the few methods of experimentelly determining the Ginzburg-Landau parameter $\kappa$ in type-I superconductors (see [10] and also [1] for other properties and developments).

P. G. de Gennes [17] (see also [22]) has proposed the formula

$$
\lim _{\kappa \rightarrow 0} \kappa\left(h^{s h}(\kappa)\right)^{2}=2^{-\frac{3}{2}} .
$$

In [3] and [5], C. Bolley and B. Helffer have rigorously proved (1.5). To get an upper bound for $A^{\prime}(0)$, these authors have proved the following estimates, for any pairs $(f, A)$ solutions of $(G L)_{\infty}$ :

$$
\begin{gathered}
A^{\prime}(0)^{2} \leq \sqrt{2}\left[\left(1-f(0)^{2}\right) f(0)^{2} \kappa^{-1}-5 A(0) f(0)^{-1}\right], \\
\kappa A^{\prime}(0)^{2} \geq \sqrt{2}\left(1-f(0)^{2}\right) f(0)^{2} .
\end{gathered}
$$

On the other hand, in [9] (see Proposition 3 p. 361), these authors have proved the following estimate: 
Proposition 1.1 There exist $\kappa_{0}$ and $C$ such that, for all $\kappa \leq \kappa_{0}$ and any solution $(f, A)$ of $(G L)_{\infty}$, we have

$$
A^{\prime}(0)^{2}=h^{2} \leq \frac{1}{2}+C \frac{f(0)}{\kappa} .
$$

From (1.6), taking the maximum of $\left(1-f(0)^{2}\right) f(0)^{2}$ on the interval $[0,1]$, and from (1.8), they have deduced the upperbound

$$
\kappa h^{2} \leq 2^{-\frac{3}{2}}+\mathcal{O}\left(\kappa^{\frac{1}{2}}\right)
$$

for all $h \in \mathcal{H}_{\infty}$ and for $\kappa$ small enough.

H. Parr [23] has proposed on the basis of some heuristic computations, the more general formula

$$
\kappa\left(h^{s h}(\kappa)\right)^{2}=2^{-\frac{3}{2}}+\frac{15}{32} \kappa+o(\kappa) .
$$

In [16], using the method of matched asymptotic expansions [21]-[26], Dorsey, Di Bartolo and Dolgert have obtained a formal expansion in powers of $\kappa^{\frac{1}{2}}$ up to order four for the superheating field $h^{s h}(\kappa)$, recovering in particular formula (1.10) at a formal level. On the other hand, in [7], C. Bolley and B. Helffer have shown that numerical computations fit very well with the Parr formula.

Constructing subsolutions and supersolutions of $(G L)_{\infty}$ based on the existence of formal solutions of the half-space Ginzburg-Landau model obtained in [16], we have proved in [13] the following theorem:

Theorem 1.2 There exist $\kappa_{0}>0$ and $C$ such that, for all $\kappa \leq \kappa_{0}$, we have

$$
\kappa\left(h^{s h}(\kappa)\right)^{2} \geq 2^{-\frac{3}{2}}+\frac{15}{32} \kappa+C \kappa^{2} .
$$

In this paper, to get a complete and rigorous proof of the Parr formula, we prove the following theorem:

Theorem 1.3 There exist $\kappa_{0}$ and $\rho>0$, such that, for all pairs $(f, A)$ solution of $(G L)_{\infty}$, for all $\kappa \leq \kappa_{0}$, we have

$$
\kappa A^{\prime}(0)^{2}=\kappa h^{2} \leq 2^{-\frac{3}{2}}+\frac{15}{32} \kappa+\mathcal{O}\left(\kappa^{1+\rho}\right) .
$$

The approach proposed by the physicists Dorsey, Di Bartolo and Dolgert and the approach exposed here are distinct. The first uses the method of asymptotic matched expansions and leads in particular to a formal proof of the Parr formula. The second one is essentially based on the maximum principle and leads to a rigorous proof of this formula. Nevertheless, we are guided in our analysis by the structure of the formal construction.

The key of Theorem 1.3 is the improvement of estimates for $f^{\prime}, A$ and $A^{\prime}$ obtained in [3] and [4]. Notably, we get an estimate for $f^{\prime}$ on $\left[0, \kappa^{-\rho}\right], \rho>0$ coinciding with the formal estimate given in [16].

The plan of this paper is the following. In Section 2, we first recall some estimates for the functions $f, A, f^{\prime}$ and $A^{\prime}$ obtained in [3] and [5]. We get new estimates for $A$ and $A^{\prime}$. In Section 3, we analyze the estimate for $A^{\prime}(0)=h$ obtained by C. Bolley and B. Helffer in [5].We get the estimate for $f^{\prime}$. In Section 4, we improve the upper bound for $A^{\prime}(0)$ given in (1.6) and get Theorem 1.3. We deduce the Parr Formula (1.10).

\section{Properties of solutions of $(G L)_{\infty}$}

\subsection{General properties of the Ginzburg-Landau equations}

Let us recall some properties of the functions $f, A, f^{\prime}$ and $A^{\prime}$ obtained in [3] and [4]. 
Proposition 2.1 Let $(f, A)$ be a solution of $(G L)_{\infty}$.

1. $f$ is increasing on $\mathbb{R}^{+}$and we have

$$
0<f(x) \leq \tanh \left(\frac{\kappa x}{\sqrt{2}}+x_{0}\right), \quad \tanh \left(x_{0}\right)=f(0) .
$$

2. A is strictly increasing on $[0,+\infty[$ and we have

$$
0 \leq A^{\prime}(x) \leq h, \quad \forall x \in[0,+\infty[.
$$

3. $f^{\prime}$ satisfies the inequalities

$$
0 \leq f^{\prime}(x) \leq \frac{\kappa}{\sqrt{2}}, \quad \forall x \in[0,+\infty[.
$$

4. The pair $(f, A)$ satisfies the following energy conservation

$$
\kappa^{-2} f^{\prime}(x)^{2}+A^{\prime}(x)^{2}=A(x)^{2} f(x)^{2}+\frac{\left(1-f(x)^{2}\right)^{2}}{2}, \quad \forall x \in[0,+\infty[.
$$

5. The pair $(f, A)$ satisfies the inequalities

$$
0<-A f \leq A^{\prime}, \quad \forall x \in[0,+\infty[
$$

Remark 2.2 From Proposition 2.1 (see (2.1)), we deduce that in a region $\left[0, \kappa^{-\rho}\right], \rho>0$, we have for some $c>0$

$$
f(0) \leq f(x) \leq f(0)+c \kappa^{1-\rho} .
$$

\subsection{New estimates for $f^{\prime}, A$ and $A^{\prime}$}

In the next sections, we use two useful versions of the maximum principle (cf. [5] and [8]).

Lemma 2.3 Let $d \in \mathbb{R}^{+} \cup\{+\infty\}$. Let $C$ be a bounded function on $] 0, d[$ such that

$$
C(x) \geq 0, \quad \forall x \in] 0, d[,
$$

and let $u \in C^{2}([0, d])$ be a function such that

$$
\left\{\begin{array}{l}
\left.-u(x)^{\prime \prime}+C(x) u(x) \leq 0 \text { on }\right] 0, d[ \\
u^{\prime}(0) \geq 0
\end{array}\right.
$$

If $d \in \mathbb{R}^{+}$, we assume that $u(d) \leq 0$, and if $d=+\infty, u^{\prime}(x) \rightarrow 0$ when $x \rightarrow+\infty$.

Then, in these two cases,

$$
u(x) \leq 0 \text { on }] 0, d[\text {. }
$$

From Proposition 1.1, we deduce that Inequality (1.12) is true for any pairs $(f, A)$ solutions of $(G L)_{\infty}$ such that $f(0) \leq \frac{1}{10 C}$. In all the following sections, we will restrict us to the pairs $(f, A)$ such that

$$
f(0) \geq \frac{1}{10 C}
$$

Some inequalities on the functions $f^{\prime}, A$ and $A^{\prime}$ are not optimal in $\left[0, \kappa^{-\rho}\right]$. In this section, we get a better control on $A$ and $A^{\prime}$. Let us recall that in [3], C. Bolley and B. Helffer have obtained the following control for $A$

$$
h \exp (-x) \leq-A \leq \frac{h}{f(0)} \exp (-f(0) x), \quad \forall x \in \mathbb{R}^{+}
$$


In [14] (see also [12] and [16]), we have constructed an asymptotic matched solution $\left(f^{v d,(n)}, A^{v d,(n)}\right)$ of $(G L)_{\infty}$. Let us recall that

$$
f^{v d,(0)}(x)=\tanh \left(\frac{\kappa x}{\sqrt{2}}+x_{0}\right)
$$

and

$$
\kappa^{\frac{1}{2}} A^{v d,(0)}(x)=-2^{\frac{1}{4}}\left(1-A_{0}^{2}\right)^{\frac{1}{2}} \exp \left(-A_{0} x\right),
$$

where $A_{0}=\tanh \left(x_{0}\right), x_{0} \geq 0$.

The comparison with the behavior of the formal solution suggests that the lower bound for $-A$ is not optimal in the interval $\left[0, \kappa^{-\rho}\right]$. We get a better lower bound for $-A$ in the following proposition:

Proposition 2.4 Let $\rho \in] 0, \frac{1}{2}\left[\right.$. There exist $\kappa_{0}>0$ and $C>0$ such that, for all $\kappa \leq \kappa_{0}$, for all pairs $(f, A)$ solutions of $(G L)_{\infty}$ satisfying (2.8), the function A satisfies the inequality

$$
\forall x \in\left[0, \kappa^{-\rho}\right], \quad-A \geq \frac{h}{f(0)}\left(1-C \kappa^{1-2 \rho}\right) \exp -(f(0) x) .
$$

\section{Proof}

We set:

$$
v_{2}(x):=\tanh \left(\frac{\kappa x}{\sqrt{2}}+x_{0}\right), \quad \tanh \left(x_{0}\right)=f(0)
$$

From (2.1), we get

$$
f \leq v_{2}
$$

Let us consider the unique solution in $H^{2}(] 0,+\infty[)$ of the problem

$$
\left\{\begin{array}{l}
-W^{\prime \prime}+v_{2}^{2} W=0 \\
W^{\prime}(0)=h
\end{array}\right.
$$

From (2.15), and applying Lemma 2.3 with $d=+\infty, C=v_{2}^{2}$ and $u=W$, we get $W \leq 0$ on $\mathbb{R}^{+}$. From $(1.3)_{b},(2.14)$ and $(2.15)$, we get $-(A-W)^{\prime \prime}+f^{2}(A-W) \leq 0$. Applying again Lemma 2.3 with $d=+\infty, C=f^{2}$ and $u=A-W$, we get

$$
A \leq W
$$

We set

$$
\phi(x):=\int_{0}^{x}\left(v_{2}+c_{1} \kappa\right) d t
$$

where $c_{1}$ is a strictly positive parameter, which will be determined later. We consider

$$
z(x)=z(0) \exp (-\phi(x))
$$

where $z(0)$ is determined by the condition $z^{\prime}(0)=h$. We get $z^{\prime}(0)=-\left(v_{2}(0)+c_{1} \kappa\right) z(0)=h$. Hence, from (2.13), it derives that

$$
z(0)=-\frac{h}{f(0)+c_{1} \kappa} .
$$

We have

$$
-(W-z)^{\prime \prime}+v_{2}^{2}(W-z)=z(0) \exp (-\phi(x))\left(-\frac{\kappa}{\sqrt{2}} \frac{1}{\cosh ^{2}\left(\frac{\kappa x}{\sqrt{2}}+x_{0}\right)}+2 v_{2} c_{1} \kappa+c_{1}^{2} \kappa^{2}\right) .
$$

From the definition of the function $v_{2}$, we get

$$
-\frac{1}{\sqrt{2}} \cosh ^{-2}\left(\frac{\kappa x}{\sqrt{2}}+x_{0}\right)+2 v_{2} c_{1}+c_{1}^{2} \kappa \geq 2 c_{1} \tanh \left(x_{0}\right)-\frac{1}{\sqrt{2}} \cosh ^{-2}\left(x_{0}\right) .
$$


We choose $c_{1}$ such that

$$
c_{1}>\frac{1}{2 \sqrt{2}}\left(\sinh \left(x_{0}\right) \cosh \left(x_{0}\right)\right)^{-1}
$$

From (2.19), as $z(0)<0$ and according to (2.20), (2.21) and (2.22), we get

$-(W-z)^{\prime \prime}+v_{2}^{2}(W-z)<0$. Applying Lemma 2.3 on $\left[0,+\infty\left[\right.\right.$ with $C=v_{2}^{2}$ and $u=W-z$, we deduce that $W-u \leq 0$. From (2.18) and (2.19), it results that

$$
-W \geq-z=\frac{h}{f(0)+c_{1} \kappa} \exp (-\phi(x)) .
$$

From (2.17), we get

$$
\phi(x)=\phi^{\prime}(0) x+x^{2} \int_{0}^{1} \phi^{\prime \prime}(\theta x) d \theta .
$$

According to (2.13) and (2.17) it derives that there exists $\rho>0$ such that

$$
\forall x \in\left[0, \kappa^{-\rho}\right], \quad \phi(x) \leq\left(f(0)+c_{1} \kappa\right) x+\frac{\kappa^{1-2 \rho}}{\sqrt{2}} .
$$

Finally, from (2.16), (2.23) and (2.24), we get the inequality

$$
-A \geq-W \geq \frac{h}{f(0)+c_{1} \kappa} \exp \left(-f(0) x-c_{1} \kappa x-\frac{\kappa^{1-2 \rho}}{\sqrt{2}}\right) .
$$

From (2.8), we deduce that there exist $C>0$ and $\kappa_{0}$, such that, for all $\left.\left.\kappa \in\right] 0, \kappa_{0}\right]$, for all $x \in\left[0, \kappa^{-\rho}\right]$, we have the inequality

$$
-A \geq \frac{h}{f(0)}\left(1-C \kappa^{1-2 \rho}\right) \exp (-f(0) x) .
$$

The proof of Proposition 2.4 follows.

We have also to get a lower bound and an upper bound for $A^{\prime}$. In [3], C. Bolley and B. Helffer get in Proposition 2.4 the following estimate for $A^{\prime}$ :

$$
h \exp (-x) \leq A^{\prime}(x) \leq \frac{h}{f(0)} \exp (-f(0) x), \quad \forall x \in \mathbb{R}^{+} .
$$

Equalities (2.10) and (2.11) suggest that these estimates are not optimal in $\left[0, \kappa^{-\rho}\right]$. We get a better control for $A^{\prime}$ in the following proposition:

Proposition 2.5 Let $\rho \in\left[0, \frac{1}{4}\right]$. There exists $\kappa_{0}>0$ such that, for all $\kappa \leq \kappa_{0}$ and for all $(f, A)$ solutions of $(G L)_{\infty}$ satisfying (2.8), we have the following estimate for $A^{\prime}$ :

$$
A^{\prime}=\left(h+\mathcal{O}\left(\kappa^{\frac{1}{2}-2 \rho}\right)\right) \exp (-f(0) x), \quad \forall x \in\left[0, \kappa^{-\rho}\right] .
$$

\section{Proof}

From $(1.3)_{b}$, the function $A^{\prime}$ satisfies

$$
-\left(A^{\prime}\right)^{\prime \prime}+f^{2} A^{\prime}=-2 f^{\prime} f A, \quad A^{\prime \prime}(0)=A(0) f(0)^{2} .
$$

From (2.1), (2.3) and (2.9), we get the inequality

$$
-2 f^{\prime} f A \leq \frac{2 \kappa h}{\sqrt{2} f(0)} \exp (-f(0) x), \quad \forall x \in \mathbb{R}^{+} .
$$


We can compare $A^{\prime}$ with the unique solution in $H^{2}\left(\mathbb{R}^{+}\right)$of

$$
\left\{\begin{array}{l}
-y^{\prime \prime}+f(0)^{2} y=\frac{\sqrt{2} \kappa h}{f(0)} \exp (-f(0) x) \\
y^{\prime}(0)=A(0) f(0)^{2}
\end{array}\right.
$$

given by

$$
y(x)=\left(-A(0) f(0)+\frac{\sqrt{2} \kappa h(1+f(0) x)}{2 f(0)^{3}}\right) \exp (-f(0) x) .
$$

According to (2.27), (2.28) and (2.29), we get $-\left(A^{\prime}-y\right)^{\prime \prime}+f^{2} A^{\prime}-f(0)^{2} y \leq 0$. Applying the principle maximum (see Lemma 2.3) with $d=+\infty, C(x)=f(0)^{2}$ and $u=A^{\prime}-y$, we get $A^{\prime} \leq y$ on $\mathbb{R}^{+}$. From (2.5) at the point $x=0$, it results that $-A(0) f(0) \leq h$. From (1.9), we have $h=\mathcal{O}\left(\kappa^{-\frac{1}{2}}\right)$. As $\frac{1}{10 C} \leq f(0) \leq 1$, for $x \in\left[0, \kappa^{-\rho}\right]$, we deduce the estimate

$$
\frac{\sqrt{2} \kappa h(1+f(0) x)}{2 f(0)^{3}}=\mathcal{O}\left(\kappa^{\frac{1}{2}-\rho}\right)
$$

As $A^{\prime} \leq y$, and from $(2.30)$, it results that

$$
A^{\prime} \leq\left(h+\mathcal{O}\left(\kappa^{\frac{1}{2}-\rho}\right)\right) \exp (-f(0) x) .
$$

To get a lower bound for $A^{\prime}$, we proceed as in Proposition 2.4. We compare $A^{\prime}$ solution of (2.27) with the unique solution in $H^{2}(] 0,+\infty[)$ of

$$
\left\{\begin{array}{l}
-W^{\prime \prime}+v_{2}^{2} W=0 \\
W^{\prime}(0)=A(0) f(0)^{2}
\end{array}\right.
$$

where $v_{2}$ is defined in (2.13). From Lemma 2.3 with $u=-W$ and $C=v_{2}^{2}$, let us remark that we have $W \geq 0$ on $\mathbb{R}^{+}$. Applying again this lemma with $d=+\infty, C=f^{2}$ and $u=W-A^{\prime}$, we deduce $W \leq A^{\prime}$. Now, we compare $W$ with the function $z$ defined in (2.18) and satisfying $z^{\prime}(0)=A(0) f(0)^{2}$. The function $z-W$ satisfies (2.20) (replacing $z(0)$ with $-z(0)$ ) and taking $c_{1}$ as in (2.22), we get $-(z-W)^{\prime \prime}+v_{2}^{2}(z-W)<0$. Applying Lemma 2.3 with $d=+\infty, u=z-W$ and $C=v_{2}^{2}$, we get

$$
z(x)=-\frac{A(0) f(0)^{2}}{f(0)+c_{1} \kappa} \exp (-\phi(x)) \leq W(x) .
$$

Following Proposition 2.4, for $x \in\left[0, \kappa^{-\rho}\right]$, we get

$$
-\frac{A(0) f(0)^{2}}{f(0)+c_{1} \kappa} \exp \left(-f(0) x-c_{1} \kappa-\frac{\kappa^{1-2 \rho}}{\sqrt{2}}\right) \leq z(x) .
$$

It derives that

$$
-A(0) f(0)\left(1-C \kappa^{1-2 \rho}\right) \exp (-f(0) x) \leq A^{\prime} .
$$

From (2.12) at the point $x=0$, we get

$$
h\left(1-C \kappa^{1-2 \rho}\right) \leq-A(0) f(0) .
$$

From (2.33), it results that

$$
h\left(1-C \kappa^{1-2 \rho}\right) \exp (-f(0) x) \leq A^{\prime} .
$$

According to (2.31) and (2.34), the proof of Proposition 2.5 follows. 


\section{Estimates for $A^{\prime}(0)$}

\subsection{Preliminaries}

First, we show that Inequality (1.12) is true for some pairs $(f, A)$ such that $f(0) \in\left[0, \frac{1}{\sqrt{2}}-\alpha \kappa^{\gamma}\right] \cup$ $\left[\frac{1}{\sqrt{2}}+\alpha \kappa^{\gamma}, 1\right],(\alpha, \gamma) \in\left(\mathbb{R}^{+}\right)^{2}$.

Lemma 3.1 There exist $\alpha>0$ and $\kappa_{0}$, such that, for all $\kappa \leq \kappa_{0}$, for all pairs $(f, A)$ solutions of $(G L)_{\infty}$ such that $\left.\left.f(0) \in\right] 0, \frac{1}{\sqrt{2}}-\alpha \kappa^{\frac{1}{4}}\right] \cup\left[\frac{1}{\sqrt{2}}+\alpha \kappa^{\frac{1}{4}}, 1\right]$, we have

$$
\kappa\left(A^{\prime}(0)\right)^{2} \leq 2^{-\frac{3}{2}}
$$

\section{Proof}

We set

$$
\phi_{0}(y)=\sqrt{2} y^{2}\left(1-y^{2}\right)
$$

This function achieves its unique maximum on $[0,1]$ at the point $y=\frac{1}{\sqrt{2}}$, and $\phi_{0}\left(\frac{1}{\sqrt{2}}\right)=2^{-\frac{3}{2}}$.

We have seen that Inequality (3.1) is satisfied for $f(0) \leq \frac{1}{10 C}$. If $f(0) \geq \frac{1}{10 C}$, then from (2.26), we have $\frac{A^{\prime}(0)}{f(0)}=\mathcal{O}\left(\kappa^{-\frac{1}{2}}\right)$. From (1.6), with the choice $f(0)=\frac{1}{\sqrt{2}}+\alpha \kappa^{\frac{1}{4}}$, we get for some $\tilde{C}$

$$
\kappa\left(A^{\prime}(0)\right)^{2} \leq 2^{-\frac{3}{2}}+\frac{1}{2} \phi_{0}^{\prime \prime}\left(\frac{1}{\sqrt{2}}\right) \alpha^{2} \kappa^{\frac{1}{2}}+\tilde{C} \kappa^{\frac{1}{2}}+\mathcal{O}_{\alpha}\left(\kappa^{\frac{3}{4}}\right) .
$$

We choose first $\alpha$ such that $\frac{1}{2} \phi_{0}^{\prime \prime}\left(\frac{1}{\sqrt{2}}\right) \alpha^{2}+\tilde{C} \leq-1$ and then $\kappa \leq \kappa_{0}$ for $\kappa_{0}$ small enough. The proof of Lemma 3.1 follows.

From now on, $\alpha$ is fixed according to Lemma 3.1 and we assume that

$$
f(0) \in\left[\frac{1}{\sqrt{2}}-\alpha \kappa^{\frac{1}{4}}, \frac{1}{\sqrt{2}}+\alpha \kappa^{\frac{1}{4}}\right], \alpha>0 .
$$

From (1.7), for all pairs $(f, A)$ solution of $(G L)_{\infty}$ satisfying (3.3), we have

$$
\kappa h^{2} \geq 2^{-\frac{3}{2}}+\mathcal{O}\left(\kappa^{\frac{1}{4}}\right) .
$$

From (1.9) and (3.4), we deduce that

$$
\kappa h^{2}=2^{-\frac{3}{2}}+\mathcal{O}\left(\kappa^{\frac{1}{4}}\right)
$$

for all pairs $(f, A)$ solution of $(G L)_{\infty}$ satisfying (3.3). In order to get Inequality (1.12), let us analyze the proof by C. Bolley and B. Helffer of the De Gennes formula [5] p. 604, and improve some of their estimates. For doing this, we are guided by the analysis of the properties of the approximate solution constructed in [14]. To get an upper bound on $A^{\prime}(0)$, C. Bolley and B. Helffer start by the identity

$$
h^{2}=-2 \int_{0}^{+\infty} A^{\prime}(t) A^{\prime \prime}(t) d t .
$$

Then, from $(1.3)_{(b)}$, they get

$$
h^{2}=2 \int_{0}^{+\infty} A^{\prime}(t)\left(-A(t) f(t)^{2}\right) d t .
$$


Then, using Inequality (2.5), they obtain

$$
h^{2} \leq 2 \int_{0}^{+\infty} f(t) A^{\prime}(t)^{2} d t
$$

We suspect that something has been lost when writing

$$
-A f \leq A^{\prime}
$$

Then, using $(1.3)_{(a)}$ and the energy conservation (2.4), they get

$$
h^{2} \leq 2 \int_{0}^{+\infty} f(t) A^{\prime}(t)^{2} d t=\int_{0}^{+\infty} f(x)\left(1-f(x)^{4}\right) d x-6 \kappa^{-2} \int_{0}^{+\infty} f(x) f^{\prime}(x)^{2} d x .
$$

In order to get the control of the right-hand side of Inequality (3.8), in particular the two terms $f^{\prime}$ and $1-f^{2}$, they use the conservation law (2.4). If we rewrite the energy conservation in the form

$$
\left(\kappa^{-1} f^{\prime}(x)+A^{\prime}(x)\right)^{2}=A(x)^{2} f(x)^{2}+2 \kappa^{-1} f^{\prime}(x) A^{\prime}(x)+\frac{1}{2}\left(1-f(x)^{2}\right)^{2},
$$

we observe that these authors have neglected the positive term $A(x)^{2} f(x)^{2}+2 \kappa^{-1} f^{\prime}(x) A^{\prime}(x)$ for getting the inequality

$$
\kappa^{-1} f^{\prime}(x)+A^{\prime}(x) \geq \frac{1}{\sqrt{2}}\left(1-f(x)^{2}\right) .
$$

To improve Inequality (3.8), in the next sections, we get an upper bound and a lower bound for the difference $\left(A^{\prime}\right)^{2}-A^{2} f^{2}$. Moreover, we improve the control on $f^{\prime}$ given in (2.3). This is the object of the following subsection.

\subsection{Estimate for $f^{\prime}$}

In order to get an estimate on $f^{\prime}$ on the interval $\left[0, \kappa^{-\rho}\right]$, we establish the following lemma:

Lemma 3.2 Let $\rho \in\left[0, \frac{1}{4}\right]$. There exists $\kappa_{0}$ such that, for all $\kappa \leq \kappa_{0}$, for all pairs $(f, A)$ solutions of $(G L)_{\infty}$ satisfying (3.3), we have the following estimate

$$
A^{\prime} f^{\prime}=\frac{\kappa^{2} h^{3}}{2 f(0)^{2}} \exp (-f(0) x)\left(1-\exp (-2 f(0) x)+\mathcal{O}\left(\kappa^{\rho}\right)\right), \quad \forall x \in\left[0, \kappa^{-\rho}\right] .
$$

\section{Proof}

Step 1: Estimate for $A^{\prime} f^{\prime}$ at the point $x=\kappa^{-\rho}$. Let $\rho \in\left[0, \frac{1}{4}\right]$. We set $Y=A^{\prime} f^{\prime}$. As $f^{\prime} \geq 0$ and $A^{\prime} \geq 0$, we have $Y \geq 0$. In order to use Lemma 2.3 with $d=\kappa^{-\rho}$, we estimate $A^{\prime} f^{\prime}$ at the point $x=\kappa^{-\rho}$. From (2.1), (2.4) at the point $x=\kappa^{-\rho}$ and (2.5), we deduce that

$$
f^{\prime}\left(\kappa^{-\rho}\right) \leq \frac{\kappa}{\sqrt{2}}\left(1-f\left(\kappa^{-\rho}\right)^{2}\right) \leq \frac{\kappa}{\sqrt{2}}\left(1-\tanh \left(x_{0}\right)^{2}\right) .
$$

According to $(2.26)$, and as $\tanh \left(x_{0}\right)=f(0)$ with $f(0) \in\left[\frac{1}{\sqrt{2}}-\alpha \kappa^{\frac{1}{4}}, \frac{1}{\sqrt{2}}+\alpha \kappa^{\frac{1}{4}}\right]$, we deduce that there exists $\hat{C}$ such that

$$
\left(A^{\prime} f^{\prime}\right)\left(\kappa^{-\rho}\right) \leq\left(\frac{\kappa h}{2 \sqrt{2}}+\hat{C} \kappa^{\frac{3}{4}}\right) \exp \left(-f(0) \kappa^{-\rho}\right) .
$$

To get a lower bound for $\left(A^{\prime} f^{\prime}\right)\left(\kappa^{-\rho}\right)$, let us remark that, from (2.9) and (2.25) at the point $x=\kappa^{-\rho}$, for $\kappa$ small, we have $\left(A^{2} f^{2}-A^{\prime 2}\right)\left(\kappa^{-\rho}\right)=\mathcal{O}\left(\kappa^{-1}\right) \exp \left(-f(0) \kappa^{-\rho}\right)=\mathcal{O}\left(\kappa^{2}\right)$. From (2.4) at the point $x=\kappa^{-\rho}$, we deduce that

$$
\kappa^{-2} f^{\prime}\left(\kappa^{-\rho}\right)^{2}=\frac{\left(1-f\left(\kappa^{-\rho}\right)^{2}\right)^{2}}{2}+\mathcal{O}\left(\kappa^{2}\right) \geq \frac{1}{2}\left(1-\tanh ^{2}\left(\frac{\kappa^{1-\rho}}{\sqrt{2}}+x_{0}\right)\right)^{2}+\mathcal{O}\left(\kappa^{2}\right) .
$$


Hence, from (3.3), we get

$$
f^{\prime}\left(\kappa^{-\rho}\right) \geq \frac{\kappa}{2 \sqrt{2}}\left(1+\mathcal{O}\left(\kappa^{\frac{3}{4}}\right)\right) .
$$

According to $(2.26)$, it results that there exists $\tilde{C}$ such that

$$
\left(\frac{\kappa h}{2 \sqrt{2}}+\tilde{C} \kappa^{\frac{3}{4}}\right) \exp \left(-f(0) \kappa^{-\rho}\right) \leq\left(A^{\prime} f^{\prime}\right)\left(\kappa^{-\rho}\right)
$$

Step 2: Upper bound for Y. Using the Ginzburg-Landau equations (1.3), we get

$$
Y^{\prime}=A f^{2} f^{\prime}+\kappa^{2} A^{\prime}\left(-f+f^{3}+A^{2} f\right) .
$$

We observe that $Y^{\prime}(0)=h f^{\prime \prime}(0)$. Differentiating once more and using (1.3), we deduce that the function $Y$ is the unique solution in $H^{2}\left(\mathbb{R}^{+}\right)$of the problem

$$
\left\{\begin{array}{l}
Y^{\prime \prime}-f^{2} Y \\
=2 f f^{\prime 2} A+2 \kappa^{2} A f^{2}\left(-f+f^{3}+A^{2} f\right)+\kappa^{2} A^{\prime}\left(-f^{\prime}+3 f^{\prime} f^{2}\right)+2 \kappa^{2} A^{\prime 2} A f+2 \kappa^{2} A^{\prime} A^{2} f^{\prime} \\
Y^{\prime}(0)=h f^{\prime \prime}(0) .
\end{array}\right.
$$

Hence, we get

$$
\left\{\begin{array}{l}
-Y^{\prime \prime}+G Y=-2 f f^{\prime 2} A-2 \kappa^{2} A f^{2}\left(f^{3}-f\right)-2 \kappa^{2} A^{3} f^{3}-2 \kappa^{2} A^{\prime 2} A f, \\
Y^{\prime}(0)=h f^{\prime \prime}(0),
\end{array}\right.
$$

where

$$
G:=f^{2}+\kappa^{2}\left(-1+3 f^{2}+A^{2}\right) .
$$

According to (2.6) and (2.9), for $x \in\left[0, \kappa^{-\rho}\right]$, we get

$$
f(0)^{2} \leq G(x) \leq f(0)^{2}+\mathcal{O}\left(\kappa^{1-\rho}\right) .
$$

In order to get an upper bound for $Y$, we look for an upper bound of the right-hand side of (3.12). First, let us analyze the main term of (3.12) which is given by $-2 \kappa^{2} A f\left(A^{2} f^{2}+A^{\prime 2}\right)$. According to (2.6), (2.9), it derives that there exists $C_{2}$ such that, for $x \in\left[0, \kappa^{-\rho}\right]$, we get

$$
-2 \kappa^{2} A f\left(A^{2} f^{2}+A^{\prime 2}\right) \leq\left(4 \kappa^{2} h^{3}+C_{2} \kappa^{1-\rho}\right) \exp (-3 f(0) x) .
$$

As $0 \leq f \leq 1$ and $A<0$ on $\mathbb{R}^{+}$, we get

$$
-2 f f^{\prime 2} A-2 \kappa^{2} A f^{2}\left(f^{3}-f\right) \leq-2 f f^{\prime 2} A .
$$

As $h=\mathcal{O}\left(\kappa^{-\frac{1}{2}}\right)$ (see (1.9)), and from (2.3), (2.9), (2.26) and (3.3), there exists $C_{1}$ such that, for all $x \in\left[0, \kappa^{-\rho}\right]$, we get

$$
-2 f f^{\prime 2} A \leq C_{1} \kappa^{\frac{3}{2}} \exp (-f(0) x) .
$$

Then, to get an upper bound for $Y$, we compare this function with the solution in $H^{2}\left(\left[0, \kappa^{-\rho}\right]\right)$ of

$$
-y_{1}^{\prime \prime}+f(0)^{2} y_{1}=\left(4 \kappa^{2} h^{3}+C_{2} \kappa^{1-\rho}\right) \exp (-3 f(0) x)+C_{1} \kappa^{\frac{3}{2}} \exp (-f(0) x)
$$

satisfying

$$
y_{1}^{\prime}(0)=Y^{\prime}(0), \quad y_{1}\left(\kappa^{-\rho}\right)=\left(\frac{\kappa h}{2 \sqrt{2}}+\hat{C} \kappa^{\frac{3}{4}}\right) \exp \left(-f(0) \kappa^{-\rho}\right) .
$$


From (3.14) and as $Y \geq 0$, we have

$$
-\left(Y-y_{1}\right)^{\prime \prime}+f(0)^{2}\left(Y-y_{1}\right) \leq-\left(Y-y_{1}\right)^{\prime \prime}+G Y-f(0)^{2} y_{1} \leq 0
$$

From (3.10) and (3.16), we have $\left(Y-y_{1}\right)^{\prime}(0)=0$ and $\left(Y-y_{1}\right)\left(\kappa^{-\rho}\right) \leq 0$. Applying Lemma 2.3 with $d=\kappa^{-\rho}, C=f(0)^{2}$ and $u=Y-y_{1}$, for $x \in\left[0, \kappa^{-\rho}\right]$, we get $Y \leq y_{1}$ on $\left[0, \kappa^{-\rho}\right]$. The solution of $(3.15)$ is given by

$$
\begin{aligned}
& y_{1}(x)=\tilde{C}_{1}(\kappa) \exp (-f(0) x)+\tilde{C}_{2}(\kappa) \exp (f(0) x) \\
& \left.-\frac{1}{8 f(0)^{2}} \exp (-3 f(0) x)\left(4 \kappa^{2} h^{3}+C_{2} \kappa^{1-\rho}\right)\right)+\exp (-f(0) x)\left(\frac{C_{1}}{4 f(0)^{2}} \kappa^{\frac{3}{2}}(1+2 f(0) x)\right) .
\end{aligned}
$$

For $x \in\left[0, \kappa^{-\rho}\right],\left(\rho \leq \frac{1}{4}\right)$, we have $\kappa^{\frac{3}{2}}(1+2 f(0) x)=\mathcal{O}(\kappa)$. Hence, from (3.16) and (3.17), we get

$$
\begin{aligned}
& y_{1}\left(\kappa^{-\rho}\right)=\tilde{C}_{2}(\kappa) \exp \left(f(0) \kappa^{-\rho}\right)+\left(\tilde{C}_{1}(\kappa)+\mathcal{O}(\kappa)\right) \exp \left(-f(0) \kappa^{-\rho}\right) \\
& =\left(\frac{\kappa h}{2 \sqrt{2}}+\hat{C} \kappa^{\frac{3}{4}}\right) \exp \left(-f(0) \kappa^{-\rho}\right) .
\end{aligned}
$$

As $\kappa h=\mathcal{O}\left(\kappa^{\frac{1}{2}}\right)$, from $(3.18)$, it results that

$$
\tilde{C}_{2}(\kappa)=\left(-\tilde{C}_{1}(\kappa)+\mathcal{O}\left(\kappa^{\frac{1}{2}}\right)\right) \exp \left(-2 f(0) \kappa^{-\rho}\right) .
$$

From (3.17), we have

$$
y_{1}^{\prime}(0)=f(0) \tilde{C}_{2}(\kappa)-f(0) \tilde{C}_{1}(\kappa)+\frac{1}{8 f(0)}\left(12 \kappa^{2} h^{3}-2 C_{1} \kappa^{\frac{3}{2}}+3 C_{2} \kappa^{1-\rho}\right) .
$$

From (3.19) and the condition $y_{1}^{\prime}(0)=Y^{\prime}(0)=h f^{\prime \prime}(0)$, we get for $\kappa$ small

$$
\tilde{C}_{1}(\kappa)=-\frac{h f^{\prime \prime}(0)}{f(0)}+\frac{3}{2} \frac{\kappa^{2} h^{3}}{f(0)^{2}}+\mathcal{O}\left(\kappa^{1-\rho}\right) .
$$

From (1.3), (2.9) and (2.12), we get

$$
f^{\prime \prime}(0)=\frac{\kappa^{2} h^{2}}{f(0)}+\mathcal{O}\left(\kappa^{2}\right)
$$

Hence, we get

$$
\tilde{C}_{1}(\kappa)=\frac{\kappa^{2} h^{3}}{2 f(0)^{2}}+\mathcal{O}\left(\kappa^{1-\rho}\right)
$$

According to (3.17) and (3.19), for $x \in\left[0, \kappa^{-\rho}\right]$, we get

$$
\begin{aligned}
& y_{1}(x)=-\frac{\kappa^{2} h^{3}}{2 f(0)^{2}} \exp (-3 f(0) x) \\
& +\exp (-f(0) x)\left[\tilde{C}_{1}(\kappa)+\left(-\tilde{C}_{1}(\kappa)+\mathcal{O}\left(\kappa^{\frac{1}{2}}\right)\right) \exp \left(2 f(0)\left(x-\kappa^{-\rho}\right)\right)\right] .
\end{aligned}
$$

From (3.21), we have $\tilde{C}_{1}(\kappa)=\mathcal{O}\left(\kappa^{\frac{1}{2}}\right)$. Let us remark that, for $x \in\left[0, \frac{\kappa^{-\rho}}{2}\right]$, for all $n \in \mathbb{N}$, we have

$$
\left(-\tilde{C}_{1}(\kappa)+\mathcal{O}\left(\kappa^{\frac{1}{2}}\right)\right) \exp \left(2 f(0)\left(x-\kappa^{-\rho}\right)\right)=\mathcal{O}\left(\kappa^{n}\right) .
$$

From (3.21) and (3.22), for $x \in\left[0, \frac{\kappa^{-\rho}}{2}\right]$, it results that

$$
Y \leq y_{1}(x)=-\frac{\kappa^{2} h^{3}}{2 f(0)^{2}} \exp (-3 f(0) x)+\left(\frac{\kappa^{2} h^{3}}{2 f(0)^{2}}+\mathcal{O}\left(\kappa^{1-\rho}\right)\right) \exp (-f(0) x) .
$$


If we make $\kappa_{0}$ smaller, we get $(3.9)$ on $\left[0, \kappa^{-\rho}\right]$.

Step 3: Lower bound for Y. According to (2.1), (2.3) and (2.9), and as $A^{\prime} \geq 0$ and $A<0$, we deduce that there exists $C_{3}$ such that, for $x \in\left[0, \kappa^{-\rho}\right]$, we we get

$$
-2 f f^{\prime 2} A+2 \kappa^{2} A f^{2}\left(f-f^{3}\right) \geq 2 \kappa^{2} A f^{2}\left(f-f^{3}\right) \geq C_{3} \kappa^{\frac{3}{2}} \exp (-f(0) x) .
$$

On the other hand, from (2.12) and (2.26), it results that there exists $C_{4}$ such that

$$
-2 \kappa^{2} A f\left(A^{2} f^{2}+A^{\prime 2}\right) \geq\left(4 \kappa^{2} h^{3}+C_{4} \kappa^{1-\rho}\right) \exp (-3 f(0) x), \quad \forall x \in\left[0, \kappa^{-\rho}\right] .
$$

From Remark 2.2, we can compare $Y$ with the solution in $H^{2}\left(\left[0, \kappa^{-\rho}\right]\right)$ of

$$
-y_{2}^{\prime \prime}+\left(f(0)^{2}+C_{5} \kappa^{1-\rho}\right) y_{2}=\left(4 \kappa^{2} h^{3}+C_{4} \kappa^{1-\rho}\right) \exp (-3 f(0) x)-C_{3} \kappa^{\frac{3}{2}} \exp (-f(0) x)
$$

satisfying the conditions

$$
y_{2}^{\prime}(0)=Y^{\prime}(0), \quad y_{2}\left(\kappa^{-\rho}\right)=\left(\frac{\kappa h}{2 \sqrt{2}}+\tilde{C} \kappa^{\frac{3}{4}}\right) \exp \left(-f(0) \kappa^{-\rho}\right) .
$$

According to (3.11), (3.24) and (3.25), Lemma 2.3 with $d=\kappa^{-\rho}, C(x)=f(0)^{2}+C_{5} \kappa^{1-\rho}$ and $u=y_{2}-Y$, we get $y_{2} \leq Y$ on $\left[0, \kappa^{-\rho}\right]$. We set $\bar{C}:=\sqrt{f(0)^{2}+C_{5} \kappa^{1-\rho}}$. The solution of (3.24) is given by

$$
y_{2}(x)=\hat{C}_{1}(\kappa) \exp (-\bar{C} x)+\hat{C}_{2}(\kappa) \exp (\bar{C} x)+F(x) \exp (-f(0) x),
$$

where

$$
F(x)=-\frac{4 \kappa^{2} h^{3} \exp (-2 f(0) x)+8 \frac{C_{3}}{C_{5}} \kappa^{\frac{1}{2}+\rho} f(0)^{2}-C_{3} \kappa^{\frac{3}{2}}+C_{4} \kappa^{1-\rho} \exp (-2 f(0) x)}{8 f(0)^{2}-C_{5} \kappa^{1-\rho}} \exp (-f(0) x) .
$$

From (3.5), we have $\kappa^{2} h^{3}=\mathcal{O}\left(\kappa^{\frac{1}{2}}\right)$, hence

$$
F\left(\kappa^{-\rho}\right)=\mathcal{O}\left(\kappa^{\frac{1}{2}+\rho}\right) .
$$

From (3.24) and (3.26) at the point $x=\kappa^{-\rho}$, it results that

$$
\hat{C}_{2}(\kappa)=-\hat{C}_{1}(\kappa) \exp \left(-2 \bar{C} \kappa^{-\rho}\right)+\mathcal{O}\left(\kappa^{\frac{1}{2}}\right) \exp \left(-(\bar{C}+f(0)) \kappa^{-\rho}\right) .
$$

From (3.26), we get

$$
y_{2}^{\prime}(0)=-\frac{8 \frac{C_{3}}{C_{5}} \kappa^{\frac{1}{2}+\rho} f(0)^{3}+12 \kappa^{2} h^{3} f(0)+3 \kappa^{1-\rho} C_{4} f(0)-f(0) C_{3} \kappa^{\frac{3}{2}}}{\left(-8 f(0)^{2}+C_{5} \kappa^{1-\rho}\right)}+\bar{C}\left(\hat{C}_{2}(\kappa)-\hat{C}_{1}(\kappa)\right) .
$$

The condition $y_{2}^{\prime}(0)=h f^{\prime \prime}(0),(3.20)$ and (3.27) lead to

$$
-\hat{C}_{1}(\kappa) \bar{C}+\frac{3 \kappa^{2} h^{3}}{2 f(0)}+\mathcal{O}\left(\kappa^{\frac{1}{2}+\rho}\right)=h f^{\prime \prime}(0)
$$

hence, as $\bar{C}=\sqrt{f(0)^{2}+C_{5} \kappa^{1-\rho}}$

$$
\hat{C}_{1}(\kappa)=-\frac{h f^{\prime \prime}(0)}{f(0)}+\frac{3 \kappa^{2} h^{3}}{2 f(0)^{2}}+\mathcal{O}\left(\kappa^{\frac{1}{2}+\rho}\right) .
$$

Following the step 1 , restrict us to $\left[0, \frac{\kappa^{-\rho}}{2}\right]$ and from (3.20), we get

$$
Y(x) \geq \frac{\kappa^{2} h^{3}}{2 f(0)^{2}} \exp (-f(0) x)\left(1-\exp (-2 f(0) x)+\mathcal{O}\left(\kappa^{\rho}\right)\right) .
$$

From (3.23) and (3.28), we deduce that there exists $\kappa_{0}$ such that, for all $\kappa \leq \kappa_{0}$, for all pairs $(f, A)$ satisfying (3.3), Estimate (3.9) is true on $\left[0, \kappa^{-\rho}\right]$. The proof of Lemma 3.2 follows.

From Lemma 3.2, we deduce an estimate for $f^{\prime}$ in the following proposition: 
Proposition 3.3 Let $\rho \in\left[0, \frac{1}{8}\right]$. There exists $\kappa_{0}$ such that, for all $\kappa \leq \kappa_{0}$, for all pairs $(f, A)$ solutions of $(G L)_{\infty}$ satisfying (3.3), we have the estimate

$$
f^{\prime}(x)=\kappa^{2} h^{2}\left(1-\exp (-\sqrt{2} x)+\mathcal{O}\left(\kappa^{\rho}\right)\right), \quad \forall x \in\left[0, \kappa^{-\rho}\right] .
$$

Proof According to Proposition 2.5 and Lemma 3.2, we get

$$
f^{\prime}(x)=\frac{\kappa^{2} h^{3}}{2 f(0)^{2}}\left(1-\exp (-2 f(0) x)+\mathcal{O}\left(\kappa^{\rho}\right)\right) \cdot\left(\frac{1}{h}+\mathcal{O}\left(\kappa^{\frac{3}{2}-2 \rho}\right)\right) .
$$

For $f(0)=\frac{1}{\sqrt{2}}+\mathcal{O}\left(\kappa^{\frac{1}{4}}\right)$, and for $x \in\left[0, \kappa^{-\rho}\right]$, we have $\exp (-2 f(0) x)=\exp (-\sqrt{2} x)+\mathcal{O}\left(\kappa^{\frac{1}{4}-\rho}\right)$. As $\rho \in\left[0, \frac{1}{8}\right]$, Estimate (3.29) follows and this achieves the proof of Proposition 3.3.

Remark 3.4 For $n=1$, at the superheating field, we have shown in [14] (see also [16]) that

$$
f^{v d,(1)}(x)=\tanh \left(\frac{\kappa x}{\sqrt{2}}+x_{0}\right)+\frac{\kappa}{4} \exp (-\sqrt{2} x),
$$

where $\tanh \left(x_{0}\right)=\frac{1}{\sqrt{2}}$. From (3.30), it results that, for $x \in\left[0, \kappa^{-\rho}\right]$, we have

$$
\left(f^{v d,(1)}\right)^{\prime}(x)=\frac{\sqrt{2}}{4} \kappa(1-\exp (-\sqrt{2} x))+\mathcal{O}\left(\kappa^{2-\rho}\right) .
$$

According to (1.7) and (1.8) with $f(0)=\frac{1}{\sqrt{2}}$, the main term of Estimate (3.29) is the one of Estimate (3.31). Therefore, we suspect that Estimate (3.29) is optimal.

\subsection{Estimate for $A^{\prime}+A f$}

To get an estimate for $A^{\prime}+A f$, we use Lemma 2.3 and estimates for $A^{\prime}, A, f^{\prime}$ and $f$ obtained in Propositions 2.4 and 2.5 .

Lemma 3.5 Let $\rho \in\left[0, \frac{1}{8}\right]$. There exists $\kappa_{0}$ such that, for all $\kappa \leq \kappa_{0}$, for all pairs $(f, A)$ solutions of $(G L)_{\infty}$ satisfying (3.3), we have the following estimate

$$
A^{\prime}+A f=\frac{1}{2} \exp \left(-\frac{1}{\sqrt{2}} x\right)\left(-\kappa^{2} h^{3} \exp (-\sqrt{2} x)-\frac{1}{8 h}+3 \kappa^{2} h^{3}+\mathcal{O}\left(\kappa^{\frac{1}{2}+\rho}\right)\right), \quad \forall x \in\left[0, \kappa^{-\rho}\right] .
$$

Proof .

Step 1: Upper bound for $A^{\prime}+A f$ We introduce the function $Z:=A^{\prime}+A f$. From (2.5), this function is positive. At the point $x=\kappa^{-\rho}$, from (2.9), (2.12) and (2.26), we deduce that there exist $\hat{C}$ and $\tilde{C}$ such that

$$
\hat{C} \kappa^{\frac{1}{2}-2 \rho} \exp \left(-f(0) \kappa^{-\rho}\right) \leq Z\left(\kappa^{-\rho}\right)=\left(A^{\prime}+A f\right)\left(\kappa^{-\rho}\right) \leq \tilde{C} \kappa^{\frac{1}{2}-2 \rho} \exp \left(-f(0) \kappa^{-\rho}\right) .
$$

From $(1.3)_{b}$, we have

$$
Z^{\prime}=A f^{2}+A^{\prime} f+A f^{\prime}
$$

Differentiating more, and using the (G.L.) equations, we get

$$
Z^{\prime \prime}=A^{\prime} f^{2}+2 A f^{\prime} f+A f^{3}+2 A^{\prime} f^{\prime}+\kappa^{2} A\left(-f+f^{3}+A^{2} f\right) .
$$

It results that the function $Z$ satisfies the equation

$$
-Z^{\prime \prime}+\left(f^{2}+2 f^{\prime}\right) Z=-\kappa^{2} A\left(-f+f^{3}+A^{2} f\right) .
$$


Let us estimate $Z^{\prime}(0)$. As $f^{\prime}(0)=0$ and from (2.4) at the point $x=0$, we get

$$
Z^{\prime}(0)=f(0)(h+A(0) f(0))=\frac{f(0)\left(1-f(0)^{2}\right)^{2}}{2(h-A(0) f(0))} .
$$

From (2.5) and (2.12) at the point $x=0$, we get $h\left(2+\mathcal{O}\left(\kappa^{\frac{3}{2}-2 \rho}\right)\right) \leq h-A(0) f(0) \leq 2 h$. It results that

$$
\frac{f(0)\left(1-f(0)^{2}\right)^{2}}{4 h} \leq Z^{\prime}(0) \leq \frac{f(0)\left(1-f(0)^{2}\right)^{2}}{4 h\left(1+\mathcal{O}\left(\kappa^{\frac{3}{2}-2 \rho}\right)\right)} .
$$

Hence, as $f(0)=\frac{1}{\sqrt{2}}+\mathcal{O}\left(\kappa^{\frac{1}{4}}\right)$, we get

$$
Z^{\prime}(0)=\frac{1}{16 \sqrt{2} h}\left(1+\mathcal{O}\left(\kappa^{\frac{1}{4}}\right)\right) .
$$

Now, we can estimate the term $-\kappa^{2} A\left(-f+f^{3}+A^{2} f\right)$. First, for $x \in \mathbb{R}^{+}$, as $A<0$ and $0 \leq f \leq 1$, we have $-\kappa^{2} A\left(-f+f^{3}\right)<0$. According to $(2.6),(2.9)$ and using $f(0)=\frac{1}{\sqrt{2}}+\mathcal{O}\left(\kappa^{\frac{1}{4}}\right)$, we deduce that there exists $C_{6}$ such that, for $x \in\left[0, \kappa^{-\rho}\right]$, we get the estimate

$$
-\kappa^{2} A^{3} f \leq\left(2 \kappa^{2} h^{3}+C_{6} \kappa^{\frac{3}{4}}\right) \exp (-3 f(0) x) .
$$

To get an upper bound for $Z$, as $f^{2}+2 f^{\prime} \geq f(0)^{2}$, we compare this function with the solution in $H^{2}\left(\left[0, \kappa^{-\rho}\right]\right)$ of

$$
-V^{\prime \prime}+f(0)^{2} V=\left(2 \kappa^{2} h^{3}+C_{6} \kappa^{\frac{3}{4}}\right) \exp (-3 f(0) x),
$$

and satisfying

$$
V^{\prime}(0)=Z^{\prime}(0), \quad V\left(\kappa^{-\rho}\right)=\tilde{C} \kappa^{\frac{1}{2}-2 \rho} \exp \left(-f(0) \kappa^{-\rho}\right) .
$$

According to (3.33), (3.34), (3.36), (3.37) and as $Z \geq 0$, we can apply Lemma 2.3 with $d=\kappa^{-\rho}$, $C=f(0)^{2}$ and $u=Z-V$ and we get $Z \leq V$. For $x \in\left[0, \kappa^{-\rho}\right]$, the solution of (3.36) is given by

$$
\begin{aligned}
& V(x) \\
& =\tilde{C}_{3}(\kappa) \exp (-f(0) x)+\tilde{C}_{4}(\kappa) \exp (f(0) x)-\left(\frac{\kappa^{2} h^{3}}{4 f(0)^{2}}+\frac{C_{6}}{8 f(0)^{2}} \kappa^{\frac{3}{4}}\right) \exp (-3 f(0) x) .
\end{aligned}
$$

From (3.37) and (3.38), we get

$$
\tilde{C}_{4}(\kappa)=\left(-\tilde{C}_{3}(\kappa)+\mathcal{O}\left(\kappa^{\frac{1}{2}-2 \rho}\right)\right) \exp \left(-2 f(0) \kappa^{-\rho}\right) .
$$

From (3.38), we deduce that

$$
V^{\prime}(0)=-f(0) \tilde{C}_{3}(\kappa)+f(0) \tilde{C}_{4}(\kappa)+\frac{3 \kappa^{2} h^{3}}{4 f(0)}+\frac{3 C_{6}}{8 f(0)} \kappa^{\frac{3}{4}} .
$$

From (3.39) and taking account the condition $V^{\prime}(0)=Z^{\prime}(0)$ (see (3.35)) and replacing $f(0)$ with $\frac{1}{\sqrt{2}}+\mathcal{O}\left(\kappa^{\frac{1}{4}}\right)$, we get

$$
\tilde{C}_{3}(\kappa)=-\frac{1}{16 h}\left(1+\mathcal{O}\left(\kappa^{\frac{1}{4}}\right)\right)+\frac{3 \kappa^{2} h^{3}}{2} .
$$

We have $\exp (-f(0) x)=\exp \left(-\frac{1}{\sqrt{2}} x\right)\left(1+\mathcal{O}\left(\kappa^{\frac{1}{4}-\rho}\right)\right)$ on $\left[0, \kappa^{-\rho}\right]$. From (3.38), (3.39) and (3.40), we get the estimate, for $x \in\left[0, \frac{\kappa^{-\rho}}{2}\right]$ and for $\kappa$ small enough

$$
Z \leq V(x)=\frac{1}{2} \exp \left(-\frac{1}{\sqrt{2}} x\right)\left(-\kappa^{2} h^{3} \exp (-\sqrt{2} x)-\frac{1}{8 h}+3 \kappa^{2} h^{3}+\mathcal{O}\left(\kappa^{\frac{3}{4}-\rho}\right)\right) .
$$


Step 2: Lower bound for $A^{\prime}+A f$

First, from (2.12), we remark that there exists $C_{7}$ such that, for $x \in\left[0, \kappa^{-\rho}\right]$,

$$
-\kappa^{2} A\left(-f+f^{3}\right) \geq C_{7} \kappa^{\frac{3}{2}} \exp (-f(0) x) .
$$

According to (2.3) and (2.6), we deduce that there exists $C_{8}$ such that $f^{2}+2 f^{\prime} \leq f(0)^{2}+C_{8} \kappa^{1-\rho}$. To get a lower bound, we compare $Z$ with the solution of

$$
\left\{\begin{array}{l}
-W^{\prime \prime}+\left(f(0)^{2}+C_{8} \kappa^{1-\rho}\right) W=\left(2 \kappa^{2} h^{3}+C_{6} \kappa^{\frac{3}{4}}\right) \exp (-3 f(0) x)+C_{7} \kappa^{\frac{3}{2}} \exp (-f(0) x), \\
W^{\prime}(0)=Z^{\prime}(0), \quad W\left(\kappa^{-\rho}\right)=\hat{C} \kappa^{\frac{1}{2}-2 \rho} \exp \left(-f(0) \kappa^{-\rho}\right) .
\end{array}\right.
$$

Following the step 1, we apply Lemma (2.3) with $d=\kappa^{-\rho}, C=\left(f(0)^{2}+C_{8} \kappa^{1-\rho}\right)$ and $u=W-Z$, and we get $W \leq Z$ on $\left[0, \kappa^{-\rho}\right]$. Following Step 2. in the proof of Lemma 3.2, one can prove that

$$
W(x)=\frac{1}{2} \exp \left(-\frac{1}{\sqrt{2}} x\right)\left(-\kappa^{2} h^{3} \exp (-\sqrt{2} x)-\frac{1}{8 h}+3 \kappa^{2} h^{3}+\mathcal{O}\left(\kappa^{\frac{1}{2}+\rho}\right)\right) \leq Z(x) .
$$

Taking account (3.41) and (3.43), we deduce that there exists $\kappa_{0}$ such that, for all $\kappa \leq \kappa_{0}$, for all pairs $(f, A)$ satisfying $(3.3)$, we get Estimate $(3.32)$ on $\left[0, \kappa^{-\rho}\right]$. The proof of Lemma 3.5 follows.

Then, from Lemma 3.5, we can improve Inequality (2.5) and get the following proposition:

Proposition 3.6 There exists $\kappa_{0}$ such that, for all $\kappa \leq \kappa_{0}$, for all pairs $(f, A)$ solutions of $(G L)_{\infty}$ satisfying (3.3), we have the following estimate

$$
-A f=A^{\prime}-B, \quad \forall x \in\left[0, \kappa^{-\rho}\right]
$$

where $B$ is defined by

$$
B(x):=\frac{1}{2} \exp \left(-\frac{1}{\sqrt{2}} x\right)\left(-\kappa^{2} h^{3} \exp (-\sqrt{2} x)-\frac{1}{8 h}+3 \kappa^{2} h^{3}+\mathcal{O}\left(\kappa^{\frac{1}{2}+\rho}\right)\right) .
$$

\subsection{Control of the function $S:=\sqrt{\left(A^{\prime}\right)^{2}-A^{2} f^{2}}$}

In all the following sections, we set

$$
S^{2}:=\left(A^{\prime}\right)^{2}-A^{2} f^{2}
$$

From Proposition 3.5, we can state the following proposition:

Proposition 3.7 Let $\rho \in\left[0, \frac{1}{8}\right]$ and $S^{2}$ be the function defined in (3.46). There exists $\kappa_{0}$ such that, for all $\kappa \leq \kappa_{0}$, for all pairs $(f, A)$ solutions of $(G L)_{\infty}$ satisfying (3.3), we have the following estimate

$$
S^{2}=\frac{1}{8}\left(-8 \kappa^{2} h^{4} \exp (-\sqrt{2} x)-1+24 \kappa^{2} h^{4}+\mathcal{O}\left(\kappa^{\rho}\right)\right) \exp (-\sqrt{2} x), \quad \forall x \in\left[0, \kappa^{-\rho}\right] .
$$

\section{Proof}

According to $(2.9),(2.12),(2.25)$ and $(2.26)$, for $x \in\left[0, \kappa^{-\rho}\right]$, we deduce the estimate

$$
A^{\prime}-A f=2\left(h+\mathcal{O}\left(\kappa^{\frac{1}{2}-2 \rho}\right)\right) \exp (-f(0) x) .
$$

From (3.48) and Lemma 3.5 (see (3.32)), we can write

$$
\begin{aligned}
& S^{2}=\left(A^{\prime}-A f\right) \cdot\left(A^{\prime}+A f\right) \\
& =\frac{1}{8}\left(-8 \kappa^{2} h^{4} \exp (-\sqrt{2} x)-1+24 \kappa^{2} h^{4}+\mathcal{O}\left(\kappa^{\rho}\right)\right) \exp (-\sqrt{2} x) .
\end{aligned}
$$

The proof of Proposition 3.7 follows. 


\section{Proof of the Parr Formula}

In this section, first, using Propositions 3.3, 3.6 and 3.7, we get Inequality (1.12) for all pairs $(f, A)$ solution of $(G L)_{\infty}$ such that $f(0) \in\left[\frac{1}{\sqrt{2}}-\alpha \kappa^{\frac{1}{4}}, \frac{1}{\sqrt{2}}+\alpha \kappa^{\frac{1}{4}}\right]$. In the following, we use the elementary equality, for $a>0$ and $b>0$

$$
\sqrt{a^{2}+b^{2}}=a+b-\frac{2 a b}{a+b+\sqrt{a^{2}+b^{2}}}
$$

\section{Proof of Inequality (1.12)}

Step 1: New estimate for $A^{\prime}(0)$ According to (3.6) and Proposition 3.6 (see (3.44)), we get the upper bound for $A^{\prime}(0)^{2}$

$$
h^{2} \leq 2 \int_{0}^{\infty} f(t) A^{\prime}(t)^{2} d t-2 \int_{0}^{\kappa^{-\rho}} A^{\prime}(t) f(t) B(t) d t
$$

where $B$ is defined in (3.45). From (3.8), we get

$$
h^{2} \leq-2 \int_{0}^{\kappa^{-\rho}} A^{\prime}(t) f(t) B(t) d t+\int_{0}^{+\infty} f(x)\left(1-f(x)^{4}\right) d x-6 \kappa^{-2} \int_{0}^{+\infty} f(x) f^{\prime}(x)^{2} d x .
$$

According to (2.4) and (4.1) with the choice $a=\kappa^{-1} f^{\prime}$ and $b=S$, it results the equality

$$
\frac{1}{\sqrt{2}}\left(1-f^{2}\right)=\kappa^{-1} f^{\prime}+S-T
$$

where $T$ is defined on $\mathbb{R}^{+}$by

$$
T(x):=\frac{2 \kappa^{-1} f^{\prime} S}{S+\kappa^{-1} f^{\prime}+\sqrt{S^{2}+\kappa^{-2}\left(f^{\prime}\right)^{2}}} .
$$

From (4.3) and following [5] (see Proposition 3.1 p. 604), we get

$$
\begin{aligned}
& \int_{0}^{\infty}\left(1-f^{4}(x)\right) f(x) d x=\frac{1}{2 \sqrt{2}} \kappa^{-1}\left(1-f(0)^{2}\right)\left(3+f(0)^{2}\right) \\
& +\sqrt{2} \int_{0}^{\infty} f(x)\left(1+f(x)^{2}\right) \cdot S(x) d x-\sqrt{2} \int_{0}^{\infty} f(x)\left(1+f(x)^{2}\right) T(x) d x
\end{aligned}
$$

and

$$
\begin{aligned}
& \int_{0}^{\infty} f(x) f^{\prime}(x)^{2} d x=\frac{\kappa}{4 \sqrt{2}}\left(1-f(0)^{2}\right)^{2}-\kappa \int_{0}^{\infty} f(x) f^{\prime}(x) S(x) d x \\
& +\kappa \int_{0}^{\infty} f(x) f^{\prime}(x) T(x) d x .
\end{aligned}
$$

Hence, from (4.2) we get

$$
h^{2} \leq \kappa^{-1} \phi_{0}(f(0))+I_{1}+I_{2}+I_{3}
$$

where $\phi_{0}$ is defined in $(3.2)$,

$$
\begin{gathered}
I_{1}:=-2 \int_{0}^{\kappa^{-\rho}} A^{\prime}(t) f(t) B(t) d t \\
I_{2}:=\sqrt{2} \int_{0}^{\infty} f(x)\left(1+f(x)^{2}\right) S(x) d x+6 \kappa^{-1} \int_{0}^{\infty} f(x) f^{\prime}(x) S(x) d x, \\
I_{3}:=-\sqrt{2} \int_{0}^{\infty} f(x)\left(1+f(x)^{2}\right) T(x) d x-6 \kappa^{-1} \int_{0}^{\infty} f(x) f^{\prime}(x) T(x) d x .
\end{gathered}
$$


For all $f(0) \in] 0,1]$, we have $\phi_{0}(f(0)) \leq 2^{-\frac{3}{2}}$. Then, we get

$$
h^{2} \leq \kappa^{-1} 2^{-\frac{3}{2}}+I_{1}+I_{2}+I_{3} .
$$

For $\rho \in] 0, \frac{1}{8}\left[\right.$, we cut the integration interval in $\left[0, \kappa^{-\rho}\right]$ and $\left[\kappa^{-\rho},+\infty\left[\right.\right.$. As the functions $f$ and $f^{\prime}$ are bounded and the function $S$ admits for upper bound and lower bound an exponential polynomial in the form of $P(x) \exp (-\sqrt{2} x)$, the integrals in (4.6) and (4.7) on the interval $\left[\kappa^{-\rho},+\infty[\right.$ are equal to $\mathcal{O}\left(\kappa^{n}\right)$ for all $n \in \mathbb{N}$. According to (2.6), (2.26) and (3.45), we get

$$
\begin{aligned}
& -2 \int_{0}^{\kappa^{-\rho}} A^{\prime}(t) f(t) B(t) d t \\
& \leq-f(0) \int_{0}^{\kappa^{-\rho}} \exp (-\sqrt{2} x)\left(\kappa^{2} h^{4} \exp (-\sqrt{2} x)-\frac{1}{8}+3 \kappa^{2} h^{4}+\mathcal{O}\left(\kappa^{\rho}\right)\right) d x .
\end{aligned}
$$

From (3.5) and as $e^{-\sqrt{2} \kappa^{-\rho}}=\mathcal{O}\left(\kappa^{n}\right)$ for $\kappa$ small, it results that

$$
I_{1} \leq-\frac{3}{32}+\mathcal{O}\left(\kappa^{\rho}\right)
$$

Step 2: Estimate for $I_{2}$ On the other hand, using Proposition 3.7, and making the scaling $u=$ $\exp (-\sqrt{2} x)$, we get

$$
\begin{aligned}
& \int_{0}^{\kappa^{-\rho}} S(x) d x \\
& =\frac{1}{4} \int_{e^{-\sqrt{2} \kappa^{-\rho}}}^{1} \frac{\sqrt{2 u-u^{2}}}{u} d u+\mathcal{O}\left(\kappa^{\rho}\right)=\left[\frac{1}{4} \sqrt{2 u-u^{2}}+\frac{1}{4} \arcsin (u-1)\right]_{e^{-\sqrt{2} \kappa^{-\rho}}}^{1}+\mathcal{O}\left(\kappa^{\rho}\right)
\end{aligned}
$$

hence, for $\kappa$ small

$$
\int_{0}^{\kappa^{-\rho}} S(x) d x=\frac{\pi}{8}+\frac{1}{4}+\mathcal{O}\left(\kappa^{\rho}\right) .
$$

From (2.6), (4.10) and Proposition 3.3 (see (3.29)), it results that

$$
6 \kappa^{-1} \int_{0}^{\kappa^{-\rho}} f(x) f^{\prime}(x) S(x) d x \leq 6 \kappa^{-1}\left(f(0)+c \kappa^{1-\rho}\right) \int_{0}^{\kappa^{-\rho}} \kappa^{2} h^{2}(1-\exp (-\sqrt{2} x)) \cdot S(x) d x .
$$

From (3.5) and making the scaling $u=\exp (-\sqrt{2} x)$, we get

$$
\begin{aligned}
& \int_{0}^{\kappa^{-\rho}}(1-\exp (-\sqrt{2} x)) \cdot S(x) d x=\frac{1}{4} \int_{e^{-\sqrt{2} \kappa^{-\rho}}}^{1} \frac{(1-u)}{u} \sqrt{2 u-u^{2}} d u+\mathcal{O}\left(\kappa^{\rho}\right) \\
& =\left[\left(\frac{3}{8}-\frac{u}{8}\right) \sqrt{2 u-u^{2}}+\frac{1}{8} \arcsin (u-1)\right]_{e^{-\sqrt{2} \kappa^{-\rho}}}^{1}+\mathcal{O}\left(\kappa^{\rho}\right),
\end{aligned}
$$

hence

$$
\int_{0}^{\kappa^{-\rho}}(1-\exp (-\sqrt{2} x)) \cdot S(x) d x=\frac{\pi}{16}+\frac{1}{4}+\mathcal{O}\left(\kappa^{\rho}\right) .
$$

From (3.3) and (3.5), we get

$$
6 \kappa^{-1} \int_{0}^{\kappa^{-\rho}} f(x) f^{\prime}(x) S(x) d x \leq \frac{3}{2}\left(\frac{\pi}{16}+\frac{1}{4}\right)+\mathcal{O}\left(\kappa^{\rho}\right)
$$

According to (2.6) and (4.10), we deduce that

$$
\sqrt{2} \int_{0}^{\kappa^{-\rho}} f(x)\left(1+f(x)^{2}\right) S(x) d x \leq \sqrt{2}\left(f(0)+c \kappa^{1-\rho}\right)\left(1+\left(f(0)+c \kappa^{1-\rho}\right)^{2}\right) \int_{0}^{\kappa^{-\rho}} S(x) d x .
$$


From (4.10), we get

$$
\sqrt{2} \int_{0}^{\kappa^{-\rho}} f(x)\left(1+f(x)^{2}\right) S(x) d x \leq \frac{3}{2}\left(\frac{\pi}{8}+\frac{1}{4}\right)+\mathcal{O}\left(\kappa^{\rho}\right) .
$$

According to (4.6), (4.11) and (4.12), we get

$$
I_{2} \leq \frac{9 \pi}{32}+\frac{3}{4}+\mathcal{O}\left(\kappa^{\rho}\right)
$$

Step 3: Estimate for $I_{3}$ From Proposition 3.3 (see (3.29)) and (3.5), on the interval $\left[0, \kappa^{-\rho}\right]$, we have

$$
T \geq U:=\frac{B}{C}
$$

where

$$
B(x):=\frac{\sqrt{2}}{2}\left(1-\exp (-\sqrt{2} x)+\mathcal{O}\left(\kappa^{\rho}\right)\right) \cdot S,
$$

and

$$
C(x):=S+\frac{\sqrt{2}}{4}\left(1-\exp (-\sqrt{2} x)+\mathcal{O}\left(\kappa^{\rho}\right)\right) . S+\sqrt{S^{2}+\frac{1}{8}\left(1-\exp (-\sqrt{2} x)+\mathcal{O}\left(\kappa^{\rho}\right)\right)^{2}} .
$$

As the functions $f^{\prime}$ and $S$ are positive on $\mathbb{R}^{+}$, from (4.4), we deduce that $T \geq 0$. Thus, from (2.6) and (4.14), we get

$$
-\sqrt{2} \int_{0}^{\infty} f(x)\left(1+f(x)^{2}\right) T(x) d x \leq-\frac{3}{2} \int_{0}^{\kappa^{-\rho}} U(x) d x+\mathcal{O}\left(\kappa^{\rho}\right) .
$$

Moreover, we have

$$
-6 \kappa^{-1} \int_{0}^{\infty} f(x) f^{\prime}(x) T(x) d x \leq-\frac{3}{2} \int_{0}^{\kappa^{-\rho}}(1-\exp (-\sqrt{2} x)) \cdot U(x) d x+\mathcal{O}\left(\kappa^{\rho}\right) .
$$

Making the scaling $u=\exp (-\sqrt{2} x)$, from (3.5), (4.14), (4.15) and (4.16), we get

$$
\int_{0}^{\kappa^{-\rho}} U(x) d x=\frac{1}{\sqrt{2}} \int_{e^{-\sqrt{2} \kappa^{-\rho}}}^{1} \frac{1}{\sqrt{2} u} \frac{\sqrt{u(2-u)}(u-1)}{u-2-\sqrt{u(2-u)}} d u+\mathcal{O}\left(\kappa^{\rho}\right),
$$

and

$$
\int_{0}^{\kappa^{-\rho}}(1-\exp (-\sqrt{2} x)) \cdot U(x) d x=\frac{1}{\sqrt{2}} \int_{e^{-\sqrt{2} \kappa^{-\rho}}}^{1}-\frac{1}{\sqrt{2} u} \frac{\sqrt{u(2-u)}(u-1)^{2}}{u-2-\sqrt{u(2-u)}} d u+\mathcal{O}\left(\kappa^{\rho}\right) .
$$

For $u \in] 0,2[$, we have

$$
\int \frac{1}{\sqrt{2} u} \frac{\sqrt{u(2-u)}(u-1)}{u-2-\sqrt{u(2-u)}} d u=\frac{\sqrt{2}}{4}\left(\arcsin (u-1)-u+\sqrt{2 u-u^{2}}\right),
$$

and

$$
\begin{aligned}
& \int-\frac{1}{\sqrt{2} u} \frac{\sqrt{u(2-u)}(u-1)^{2}}{u-2-\sqrt{u(2-u)}} d u \\
& =\frac{1}{8} \arcsin (u-1)+\frac{\sqrt{2}}{8} \sqrt{2 u-u^{2}}(3-u)-\frac{\sqrt{2}}{4} u\left(1-\frac{u}{2}\right) .
\end{aligned}
$$

From (4.19), it results that

$$
\int_{0}^{\kappa^{-\rho}} U(x) d x=\frac{\pi}{8}+\mathcal{O}\left(\kappa^{\rho}\right)
$$


Moreover, from (4.20), we get

$$
\int_{0}^{\kappa^{-\rho}}(1-\exp (-\sqrt{2} x)) \cdot U(x) d x=\frac{1}{8}\left(1+\frac{\pi}{2}\right)+\mathcal{O}\left(\kappa^{\rho}\right) .
$$

From (4.17), (4.18), (4.21) and (4.22), we get

$$
I_{3} \leq-\frac{9 \pi}{32}-\frac{3}{16}+\mathcal{O}\left(\kappa^{\rho}\right)
$$

Step 4: Upper bound for $A^{\prime}(0)$

According to (4.8), (4.9), (4.13) and (4.23), we deduce that there exists $\kappa_{0}$, such that, for all $\kappa \leq \kappa_{0}$ and all pairs $(f, A)$ solutions of $(G L)_{\infty}$ satisfying $(3.3)$, we have

$$
h^{2} \leq \kappa^{-1} 2^{-\frac{3}{2}}+\frac{15}{32}+\mathcal{O}\left(\kappa^{\rho}\right) .
$$

From Lemma 3.1 and (4.24), the proof of Theorem 1.3 follows.

According to (1.11) and Theorem 1.3, we deduce the Parr Formula (1.10).

Theorem 4.1 (Parr Formula) There exists $\kappa_{0}$ such that, for all $\kappa_{0} \leq \kappa$, we have

$$
\kappa\left(h^{s h}(\kappa)\right)^{2}=2^{-\frac{3}{2}}+\frac{15}{32} \kappa+o(\kappa) .
$$

Remark 4.2 In [2], C. Bolley and the author have proved that the set $\left.\left\{\left(f_{0}, h\right) \in\right] 0,1\right] \times\left[0,+\infty\left[\right.\right.$ s.t. $\exists(f, A)$ solution of $(G L)_{\infty}$ with $\left.f(0)=f_{0}\right\}$ is a graph of a map $\sigma$ from ] $0,1]$ into $[0,+\infty[$. From Theorem 1.3, we deduce an upper bound for the maximum of $\sigma$ on $] 0,1]$.

\section{Conclusion}

In the weak- $\kappa$ limit, we have rigorously proved that the second term in the expansion of $\kappa^{\frac{1}{2}} h^{s h}(\kappa)$ is of order of $\mathcal{O}(\kappa)$ and we have recovered the constant of Parr. In [23], this author associated the initial condition $f_{0}=\frac{1}{\sqrt{2}}-\frac{7}{32} \kappa$ to the superheating field. An open problem is to prove that the second term in the expansion of $f_{0}$ is of the order of $\mathcal{O}(\kappa)$. Consequently, we will prove the following conjecture introduced in [14] (see also [24]). At the superheating field, there exists $\kappa_{0}$ such that, for all $\kappa \leq \kappa_{0}$, we have the asymptotic expansion

$$
-\frac{A_{\kappa}(0)}{A_{\kappa}^{\prime}(0)}=\sqrt{2}+\frac{3}{16} \kappa+\mathcal{O}\left(\kappa^{2}\right)
$$

More generally, in [14] (see also [16]), as a consequence of the construction of an asymptotic matched solution, we have obtained a complete expansion for the superheating field, denoted by $h^{s h, f}(\kappa)=$ $\kappa^{-\frac{1}{2}} \sum_{i=0}^{\infty} h_{i} \kappa^{i}$. In [15], we have rigorously proved that for all $n \in \mathbb{N}$, there exist $\kappa_{0}$ and $C$ such that, for all $\kappa \leq \kappa_{0}$, we have

$$
\kappa^{\frac{1}{2}} h^{s h}(\kappa) \geq \sum_{i=0}^{n} h_{i} \kappa^{i}+C \kappa^{n+1} .
$$

An open problem is to prove that for all $n \in \mathbb{N}$, we have

$$
\kappa^{\frac{1}{2}} h^{s h}(\kappa)=\sum_{i=0}^{n} h_{i} \kappa^{i}+o\left(\kappa^{n}\right) .
$$


It seems difficult to extend the approach presented here to obtain the coefficients of higher order terms. It is necessary to recover the asymptotic matched solution at all orders constructed in [14] to get these terms.

In the large- $\kappa$ limit, using a method of matched asymptotic expansions, S. Chapman [11] (see also [6] and [20]) has formally proved the formula

$$
h^{s h}(\kappa)=\frac{1}{\sqrt{2}}+C \kappa^{-\frac{4}{3}}+o\left(\kappa^{-\frac{4}{3}}\right)
$$

for some $C \sim 0.3$. This suggests that the superheating field $h^{s h}(\kappa)$ admits an expansion in powers of $\kappa^{-\frac{4}{3}}$ when $\kappa$ is large. The rigorous proof of formula (5.3) is also an open problem.

\section{Acknowledgements}

The author would like to thank C. Bolley and B. Helffer for helpful discussions on the problem.

\section{References}

[1] Aftalion, A. and Troy W.C. "On the solutions of the one-dimensional Ginzburg-Landau equations for superconductivity," Physica D 132, 331-345 (1999).

[2] Bolley, C. and Del Castillo, P., "Existence and uniqueness for the half-space Ginzburg-Landau model," Nonlin. anal. 47/1, 135-146 (2001).

[3] Bolley, C. and Helffer, B., "Rigorous results for the Ginzburg-Landau equations associated to a superconducting film in the weak $\kappa$-limit," Rev. Math. Phys. 8, 43-83 (1996).

[4] Bolley, C. and Helffer, B., "Rigorous results on the Ginzburg-Landau models in a film submitted to an exterior parallel magnetic field. Part II," Nonlinear Studies 3, 1-32 (1996).

[5] Bolley, C. and Helffer, B., "Proof of the De Gennes formula for the superheating field in the weak $\kappa$ limit," Ann. Inst. H. Poincaré, Anal. Non Linéaire 14, 597-613 (1997).

[6] Bolley, C. and Helffer, B., "The Ginzburg-Landau equations in a semi-infinite superconducting film in the large $\kappa$-limit," Eur. J. Appl. Math. 8, 347-367 (1997).

[7] Bolley, C. and Helffer, B., "Superheating in a semi-infinite film in the weak $\kappa$ limit: numerical results and approximate models," Math. Modell. Numer. Anal. 31, 121-165 (1997).

[8] Bolley, C., Foucher F., and Helffer, B., "Superheating field for the Ginzburg-Landau equations in the case of a large bounded interval," J. Math. Phys. 41, 7263-7289 (2000).

[9] Bolley, C. and Helffer, B., "A priori estimates for Ginzburg-Landau solutions," Nonlinear PDE's in Condensed Matter and Reactive Flows, NATO Science Series 569, 355-373 (2002).

[10] Burger, J.P., and Saint-James, D., "Superconductivity", R. D. Parks 2, (M. Dekker, New York, 1969).

[11] Chapman, S., "Superheating field of type II superconductors," Siam J. Appl. Math. 55, 1233-1258 (1995).

[12] Del Castillo, P., Thèse, Université Paris Sud, (2000).

[13] Del Castillo, P., "Two terms in the lower bound for the superheating field in a semi-infinite film in the weak- $\kappa$ limit," Eur. J. Appl. Math. 13, 519-547 (2002).

[14] Del Castillo, P., "Expansion for the superheating field in a semi-infinite film in the weak- $\kappa$ limit," Math. Modell. Numer. Anal. 36, 971-993 (2002). 
[15] Del Castillo, P., "Lower bound for the superheating field in a semi-infinite film in the weak- $\kappa$ limit: the general case," J. Math. Phys. 44, 2416-2450 (2003).

[16] Dolgert, A.J., Di Bartolo, S.J. and Dorsey, A.T., "Superheating fields of superconductors Asymptotic analysis and numerical results," Phys. Rev. B 53, 5650-5660 (1996); “ Erratum: Superheating fields of superconductors Asymptotic analysis and numerical results [Phys. Rev. B 53 5650 (1996)]," Phys. Rev. B 56, 2883 (1997).

[17] De Gennes, P.G., "Superconductivity: selected topics in solid state physics and theoretical Physics," Proceedings of 8th Latin american school of physics, Caracas, (1966).

[18] Ginzburg, V.L., "On the theory of superconductivity," Nuovo Cimento 2, 1234 (1955).

[19] Ginzburg, V.L., "On the destruction and the onset of superconductivity in a magnetic field," Zh. Eksp. Teor. Fiz. 34, 113-125 Transl. [Sov. Phys. JETP 7, 78-87 (1958)].

[20] Helffer, B., and Weissler, F., "On a family of solutions of the second Painlevé equation related to superconductivity," Eur. J. Appl. Math. 9, 223-243 (1998).

[21] Kaplun, S., Fluid mechanics and Singular Perturbations, (Academic, New York, 1967)

[22] The Orsay group, "In Quantum fluids," (ed. D.F. Brewer), p. 26, Amsterdam, North Holland (1966).

[23] Parr, H., "Superconductive superheating field for finite $\kappa$," Z. Physik B 25, 359-361 (1976).

[24] Rothberg-Bibby, B., Fink, H.J., and McLachlan, D.S., "First and second order phase transitions of moderately small superconductor in a magnetic field," VIIB, North-Holland, (1978).

[25] Tinkham, M., Introduction to superconductivity, (McGraw-Hill, New-York, 1975).

[26] Van Dyke, M., "Perturbation Methods in fluid mechanics," (Academic, Stanford, CA, 1975). 